$\Rightarrow$ FLUID DYNAMICS

\section{Bubbly without gravity}

Bubbles float to the top of a liquid (pictured). This buoyancy means that a cloud of bubbles disperses, usually within a few hundred milliseconds. However, buoyancy is gravity-driven and thus is lower in space than on Earth - in low-gravity environments, bubbles do not float

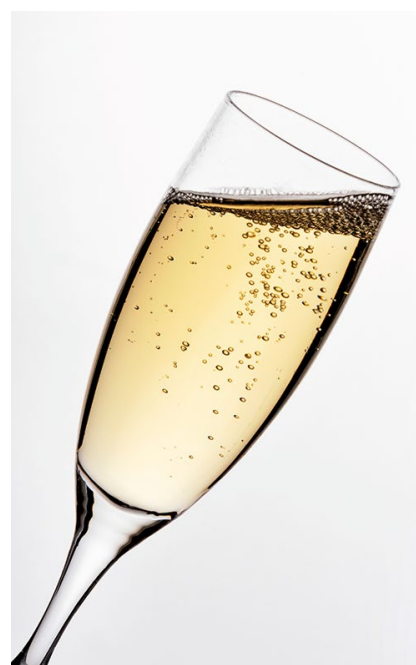

away so quickly. Understanding how bubble clouds grow in such conditions could help explain phenomena such as the loss of helium in meteorites, and enable certain technologies in space, such as solvent extraction, which is more efficient when bubbles are present.

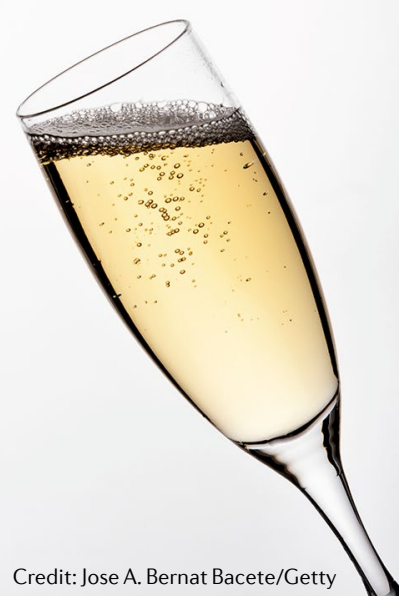

Writing in Soft Matter, Patricia Vega Martínez and colleagues report running a bubble nucleation and growth experiment in freefall in a $120 \mathrm{~m}$ drop tower, which allowed them to observe bubble clouds, unaffected by buoyancy, for several seconds.

An electrical spark in a chamber filled with carbonated water started the bubble nucleation. At first, each bubble grew independently, but as they became larger, bubbles started to interact and compete for the $\mathrm{CO}_{2}$ in the water, slowing their growth. At later times, $\mathrm{CO}_{2}$ was only available to bubbles on the outside of the cloud, and those in the interior stopped growing. In contrast, bubble clouds nucleated under Earth's gravity float away before entering this final regime. These results were corroborated by numerical simulations of a simplified model for the bubble interactions.

Zoe Budrikis

ORIGINAL ARTICLE Vega Martínez, P., Rodriguez Rodriguez, J. \& van der Meer, D. Growth of a bubble cloud in $\mathrm{CO} 2$-saturated water in microgravity.

Soft Matter https://doi.org/10.1039/D0SM00015A (2020) 